\title{
Hypochloremic metabolic alkalosis and failure to thrive: question
}

\author{
Uwe Querfeld • Silvia Lechner • Andreas R. Janecke
}

Received: 16 August 2010 /Revised: 10 September 2010 /Accepted: 16 September 2010 /Published online: 28 October 2010

(C) IPNA 2010

\section{Case summary}

The patient was born at 36 weeks of gestation with a birth weight of $2,850 \mathrm{~g}$ as the first living male child of consanguineous apparently healthy parents from Libya. The pregnancy had been complicated by polyhydramnios. The mother reported two previous pregnancies, one resulting in early abortion, and the other in an anencephalic neonate. After birth, the patient showed prolonged jaundice, vomiting, and dehydration with hypokalemia, and the clinical diagnosis of neonatal Bartter syndrome was made. Initial treatment included intravenous fluids to correct hypovolemia and oral potassium solutions. The further clinical course was characterized by persistent diarrhea (8-9/day stools daily) complicated by frequent episodes of dehydration and waterelectrolyte-imbalances leading to repeated hospitalizations. Because of the unremitting course and progressive failure to thrive, it was decided to refer the patient to our institution for further diagnosis and therapy.

A review of previous hospital records showed that the patient had needed daily potassium supplements. Since the

The answer to this question can be found at http://dx.doi.org/10.007/ s00467-010-1667-y.

U. Querfeld $(\bowtie)$

Department of Pediatric Nephrology, Charité Berlin,

Augustenburger Platz 1,

13353 Berlin, Germany

e-mail: uwe.querfeld@charite.de

\section{S. Lechner}

Division of Human Genetics, Innsbruck Medical University, Innsbruck, Austria

A. R. Janecke

Department of Pediatrics II, Innsbruck Medical University, Innsbruck, Austria child strongly disliked the salty flavor of potassium chloride, an oral potassium gluconate solution had been given at a dose of $8 \mathrm{mmol} / \mathrm{kg} /$ day. Medication and feeding had remained difficult and the child had never developed normal eating patterns, resulting in a dependence on continuous oral feeding by relatives with occasional intravenous alimentation; however, application by a nasogastric tube had been refused by the parents. Celiac disease had been excluded by intestinal biopsy. Medication with omeprazole and domperidone was without effect. Altogether, the child had spent almost half of his life in hospitals.

Upon admission, at the age of 2 years, the child appeared severely malnourished and weight $(8,900 \mathrm{~g})$, height $(81.5 \mathrm{~cm})$, and head circumference $(45 \mathrm{~cm})$ were far below appropriate percentiles for his age. Apart from abdominal distension and paleness, clinical examination revealed no further abnormalities and no congenital malformations. Blood gas analysis showed severe metabolic alkalosis $(\mathrm{pH} 7.58$, bicarbonate $46 \mathrm{mmol} / \mathrm{l}$, and base excess $+21 \mathrm{mmol} / \mathrm{l})$. Serum electrolytes were as follows: potassium $2.0 \mathrm{mmol} / \mathrm{l}$, sodium $131 \mathrm{mmol} / \mathrm{l}$, and chloride $68 \mathrm{mmol} / \mathrm{l}$. Further clinical observation after rehydration and during a period of minimal intravenous fluid replacement showed that the patient had a spontaneous total caloric intake of approximately $20 \%$ of his recommended dietary allowance and a spontaneous fluid intake of about $200 \mathrm{ml}$.

\section{Questions}

1. Do you agree with the clinical diagnosis of congenital Bartter syndrome?

2. If not, what is the diagnosis?

3. What diagnostic tests would you perform?

4. What is the clinical management of this problem? 